\title{
Walter Benjamin und die Zahl. Anmerkungen zum Ver- hältnis von mathematisch-logischer Denkform und essayistischer Schreibweise bei Walter Benjamin
}

\begin{abstract}
W filozofii Waltera Benjamina można zaobserwować tendencję do wzajemnego zmieniania się i zachodzenia na siebie dwóch jedności - jedności liczby (jako tożsamości pojęcia) i jedności konfiguracji (jako obrazu). Konsekwencją tego jest okoliczność, iż Benjamin - jak zauważył już Adorno - zamiast ,wypowiedzi zobowiązującej‘ ustanawia wypowiedź metaforyczną, iż mówi o pojęciu, lecz czyni to na sposób obrazu. Niniejszy artykuł wykazuje, iż niedostateczność oddzielenia mitu i historii, krytyki techniki i oczarowania przestrzenią meta-techniczną, która wydaje się być intencją Benjamina, ma swe źródło w jego ambiwalentnym stosunku do liczby (jako koniecznej granicy).

In der Philosophie Walter Benjamins lässt sich eine Tendenz zu einem Ineinanderumschlagen und Sich-Übereinanderschieben zweier Einheiten beobachten - der Einheit der Zahl (als Begriffsidentität) und der Einheit der Konfiguration (als Bild). Die Folge davon ist der Umstand, dass Benjamin - wie bereits Adorno bemerkt hat - anstelle der ,verpflichtenden Aussage" eine metaphorische setzt, dass er über einen Begriff spricht, jedoch im Modus des Bildes. Im vorliegenden Artikel wird aufgezeigt, dass der von Benjamin, wie es scheint, intendierten Unzulänglichkeit der Trennung von Mythos und Geschichte, der Kritik am Technischen und einer Faszination für das Meta-Technische, sein ambivalentes Verhältnis zur Zahl (als der unbedingten Grenze) zugrunde liegt.
\end{abstract}

A perceptible tendency in Walter Benjamin's philosophy is the mutual substitution and overlapping of the two distinct unities - that of the number (as an identity of idea) and that of configuration (as a picture). Therefore, as already Adorno observed, Benjamin in lieu of a ,binding statement" postulates a figurative statement, for even though he talks of an idea, he presents it in visual terms, as a picture. The paper aims to demonstrate that lack of a sufficiently precise distinction between myth and history, 
criticism of technique and fascination with meta-technical space - which appears to be Benjamin's intention - has its source in his ambivalent attitude to the number (as an indispensable boundary).

„Wenn ich sage: ich sehe das da, so ist damit nicht eine Gleichung zwischen mir und der Sache niedergelegt... Im Traume dagegen liegt eine Gleichung vor. Die Dinge, die ich sehe, sehen mich ebensowohl wie ich sie sehe." Die Worte Paul Valérys, die BENJAMIN (1972b:647) in seiner Baudelaire-Arbeit zitiert, inkludieren eine spezifische Wahrnehmungsweise der Zahl, die den streng logisch-mathematischen Verwendungsbereich verlässt und als eine optische Denkfigur der Gleichung in die Dimension des Traumhaften übergeht. Die Folge davon ist, dass die Zahl ihren rein objektiv-formalen Charakter ${ }^{1}$ verliert und - gewissermaßen außerhalb der mathematisch-logischen Verifizierbarkeit - mit Elementen Verbindungen eingeht, die durch das Differente und Heterogene gekennzeichnet sind. Diese Auffassung von der Zahl ist es auch, der wir in exemplarischer Form im Werk Walter Benjamins begegnen. Was mich nun in den folgenden Überlegungen interessiert, ist die Frage nach den Voraussetzungen, die den Geltungsbereichen von Zahl und Nichtzahl zugrunde liegen, sowie die Erhellung der Relationen, die bei Benjamin zwischen den besagten Bereichen bestehen.

Durch die extrem gesetzten Antinomien von Identität und Differenz, Vielheit und Einheit, Zentrum und Peripherie, die das Denken Benjamins kennzeichnen $^{2}$, scheinen sich die Stellen, die bei ihm die Zahl und die Nichtzahl beset-

1 Dass der Prozess der Formalisierung der Zahl mit der Geburt der neuzeitlichen Naturwissenschaft zusammengeht, bereits bei Galileo einsetzt und bei Newton und Leibniz kulminiert, liegt auf der Hand. Die Zahl trennt sich damit von jeglicher magischen, ästhetischen oder ethischen Zuordnung, haftet nicht mehr an den Dingen und wird somit inhaltslos. Auf dem Gebiet der modernen Logik entspricht dies dem Ansatz Freges und Russels. Die Zahlen lassen sich nach ihnen ,[...] nicht als Eigenschaften von Dingen verstehen, so wie etwa die Qualität ,Rotsein' Einzeldingen oder auch einer Anzahl von Dingen zugesprochen werden kann. Zahl ist vielmehr eine Eigenschaft, die erst dem Begriff einer Menge von Dingen zugesprochen werden kann; z. B. die Zahl 2 der Menge aller Paare, die 3 der Menge aller Tripel usw. Der Zahlbegriff wird hier also mit Hilfe der Äquivalenzrelation zwischen Mengen definiert. Von einer Menge wird nur eine sie eindeutig definierende Eigenschaft verlangt, so daß von jedem Element feststeht, ob es zur Menge gehört oder nicht." (SCHÄFER 1974:1778)

2 Diese scharfen Antinomien hat Benjamin einem Gestaltungsprozess unterzogen, dessen Ergebnis eine Art der Verschmelzung von Bild und Begriff, von Gedichtetem und Gedachtem darstellt, die sein Werk kennzeichnet. Dem Gestaltungsvorgang lag das zugrunde, was Benjamin „Idee“ nannte - ein belebendes und 
zen, dem interpretatorischen Zugriff zu entziehen. Im Unterschied zu Autoren wie Kassner, Musil, Spengler und dann auch Flusser ${ }^{3}$, die die Ergebnisse ihrer naturwissenschaftlich-mathematischen Studien in ihr essayistisches Werk mit hineingenommen haben, hat er weder den Begriff der Zahl zu einem exponierten Gegenstand seiner Kritik gemacht noch logisch-mathematische Fragestellungen in Form einer längeren Prosaarbeit behandelt. Und dennoch scheint das Verhältnis Benjamins zur Zahl insofern bedeutsam zu sein, als es in signifikanter Weise ein Stadium auf einer Entwicklungslinie anzeigt, die die Auffassung der Zahl - außerhalb ihrer streng wissenschaftlich-mathematischen oder philosophischen Instrumentierung - in der deutschsprachigen essayistischen Prosa des 20. Jhd.s in Form von ästhetisch-semantischen Repräsentationen aufweist. ${ }^{4}$

Es seien eingangs zwei Bemerkungen vorausgeschickt, die die Grundrichtung nachfolgender Überlegungen klarstellen: Zum einen geht es darin nicht um eine Übertragung logisch-mathematischer Kategorien auf die Werke Benjamins, um sie mit den von Habermas stipulierten ,unbilligen Konsistenzforderungen“ (HABERMAS 1981:338) zu konfrontieren. Zum anderen ist festzuhalten, dass der Forderung nach einer reinen, logisch-mathematischen Text-

zentrierendes Organ, aus dem jedoch keine längeren Prosaformen (von einigen wenigen Ausnahmen abgesehen) hervorgegangen sind: „Als Gestaltung des $\mathrm{Zu}$ sammenhangs, in dem das Einmalig-Extreme mit seinesgleichen steht, ist die Idee umschrieben [...]. Vom Extremen geht der Begriff aus. Wie die Mutter aus voller Kraft sichtlich erst da zu leben beginnt, wo der Kreis ihrer Kinder aus dem Gefühl ihrer Nähe sich um sie schließt, so treten die Ideen ins Leben erst, wo die Extreme sich um sie versammeln.“ (BENJAMIN 1972e:215).

3 Obwohl Vilém Flusser nicht als kanonischer Repräsentant der deutschen Essayistik gilt, sind seine essayistischen Schriften doch exemplarisch für die Kritikform, die sich in der zweiten Hälfte des 20. Jhd.s in der Auseinandersetzung mit den neuen Medien entwickelt hat.

4 Eine noch nicht vorhandene Geschichte der Auffassung von der Zahl innerhalb der deutschen Essayistik des 20. Jhd.s könnte als in drei Phasen verlaufende dargestellt werden: In einer ersten Phase fungiert die Zahl noch als ein Polarisationsbegriff der organisch-physiognomischen Weltdeutung (Kassner); in einer zweiten Phase gewinnt sie durch die Wirkung moderner Medien (Photographie, Film) an Mehrdeutigkeit: Die Zahl steht hier für den Verlust des Auratischen und zugleich für eine neue Faszination für die Technik (Benjamin). In einer dritten Phase schließlich wird die Zahl als ubiquitär und nicht positionierbar begriffen. Damit wird sie zu einer rein funktionellen Einheit, die dem Hybriden von Abstraktion und Konkretion innerhalb der von digitalen Medienbildern vorprogrammierten Wahrnehmung zugrunde liegt (Flusser). 
struktur ein idealtypisches Konstrukt zugrunde liegt, was auch den Worten FRANZ WIEDMANNs (1988:115) entsprechen mag, dass „,selbst das begrifflich-logische Denken nicht ohne bildhafte Ausdrucksweise ist". Das hier durchgeführte Verfahren - dessen Ziel ich nun noch deutlicher formulieren möchte - zielt darauf, die Voraussetzungen freizulegen, die den Relationen zwischen den ästhetisch-semantischen Zahlrepräsentationen ${ }^{5}$ bei Benjamin (z.B. als Addition, Häufung im allegorischen Bild, als photographische oder filmtechnische Fragmentierung, als Wiederkehr alles Gleichen im Mythos usw.) und der mathematisch-logischen Verwendungsweise der Zahl zugrunde liegen.

Benjamin scheint auf das Diskontinuierliche und Disparate seiner Denkrichtung in Hinblick auf die Zahl hindeuten zu wollen, wenn er schreibt:

Was für die anderen Abweichungen sind, das sind für mich die Daten, die meinen Kurs bestimmen. - Auf den Differentialen der Zeit, die für die anderen die ,großen Linien' der Untersuchung stören, baue ich meine Rechnung auf. (BENJAMIN 1972:570)

Mit dieser Denkweise Benjamins, die das Lineare und Integrale aufbrechen will, hängt eine Schwierigkeit zusammen, auf die bereits ADORNO (1970:17) hingewiesen hat:

So gesättigt ist dies Denken mit Kultur als seinem Naturgegenstand, daß es der Verdinglichung sich verschwört, anstatt ihr unentwegt zu widersprechen. Das ist der Ursprung von Benjamins Neigung, seine geistige Kraft ans ganz Entgegengesetzte zu zedieren, wie sie in der Arbeit über das Kunstwerk im Zeitalter seiner technischen Reproduzierbarkeit den extremen Ausdruck fand. Der Blick seiner Philosophie ist medusisch.

Diesen polaren Magnetismus, dem die Denkenergie Benjamins unterworfen ist, hat Anna Freud - wie Adorno an einer anderen Stelle bemerkt - als „Identifikation mit dem Angreifer“ bezeichnet (ADORNO 1970:58). Es sei jene Verfallenheit ans Objekt, bis zur buchstäblichen Auslöschung des Selbst, kraft deren nur die Einsichten der Einbahnstraße zu erringen gewesen seien. Abweichung und Identifikation sind danach die zwei Denkbewegungen, an denen sich die Zahl bei Benjamin als Funktionsbegriff des Quantitativen, als das scharf abgrenzende Organisationsprinzip des Denkens bricht und als solche in die früher erwähnten semantisch-ästhetischen Zahlrepräsentationen eingeht.

5 In der Sprache Benjamins sind diese immer ein Ergebnis der Polarisation von bildhaften und begrifflichen Darstellungsmodi. Im Folgenden wird darauf noch zurückzukommen sein. 
Mit der programmatischen Ausrichtung der Prosa Benjamins auf das Ephemere und Unbeachtete, auf die miniaturhaften Utensilien des kulturellen Abfalls, kurz, auf all das, was sich dem intentionalen Blick entzieht, geht ein sonderbarer Verdeckungsmechanismus einher, der auch die von Benjamin scheinbar deutlich konturierten Begriffe wie Allegorie, Kunstwerk, Sprache oder Geschichte in einer sich immer neu einstellenden Gebrochenheit und Vorläufigkeit hervortreten lässt. Behandelt er in weiten Teilen des vornehmlich auf Begriffsabgrenzung und Differenzierung angelegten TrauerspielTraktats Kategorien wie die Allegorie, das Trauerspiel oder das Kunstwerk, so verzichtet er in seinen späteren Schriften nahezu ganz auf Versuche, Kategorien analytisch-begrifflich neu zu fundieren und zu entfalten, um das Verständnis des Lesers zu erleichtern. Dies tritt noch deutlicher zutage, wenn Benjamin Kategorien und Begriffe verwendet - wie die der Reproduzierbarkeit, der Wiederholung, des Gleichartigen oder der Ähnlichkeit -, die im weit gefassten Sinne dem Einzugsbereich des Quantitativ-Zahlhaften angehören. Dieser ausgeprägt monadenhaft-statische und fragmentarische Charakter der Werke Benjamins schafft Verhältnisse, unter denen die Streuung von Zahlund Nichtzahl-Bereichen sehr ungleichmäßig und inselhaft verläuft und deswegen nur in einem begrenzten Maße aus einem unmittelbareren Rückgriff auf den sachbezogenen Gedankengehalt seiner Schriften erschlossen werden kann. Im Folgenden versuche ich, die früher erwähnten Darstellungsmodi bei Benjamin in einen logisch-mathematischen Verstehenszusammenhang einzufügen, indem ich im Anschluss an Frege und Wittgenstein die Grenzpunkte der Geltungsbereiche zweier Begriffe abstecke: der Zahl und der Differenz.

Es sind zwei logisch-mathematische Grundoperationen zu statuieren, die der Zahlfunktion ${ }^{6}$ zugrunde liegen: Der Operation der ,präzisierenden Eingrenzung' entspricht der Aspekt der Eindeutigkeit und Exaktheit; der Operation der ,isolierenden Abgrenzung ' ist der Begriff der Differenz zuzuordnen. Die erste entspricht der Forderung GOTTLOB FREGEs (1971:133) nach der scharfen Begrenzung der Begriffe:

Von einem Begriffe müssen wir die scharfe Begrenzung verlangen. Das soll heißen: von jedem Gegenstand muß gelten, entweder daß er unter den Begriff falle

6 Sie setzt immer - sei es im positiven Sinne einer zweckorientierten wissenschaftlichen Explikation, sei es im negativen Sinne der Annahme, dass nur ein Denken in scharf begrenzten Begriffen Erkenntniswert beanspruchen könne eine Eingrenzung und eine Abgrenzung voraus. 
Sławomir Leśniak

oder daß er nicht unter ihn falle. Ein dritter Fall, etwa der der Unentschiedenheit oder Unbestimmtheit, darf nicht vorkommen.

Die Eindeutigkeit der logischen Distinktion, die dabei angestrebt wird, wird durch die Sätze vom ausgeschlossenen Dritten und vom ausgeschlossenen Widerspruch gewährleistet, die aus der logischen Ordnung entweder/oder resultieren. Das Denken vollzieht sich hierbei als eine Vereinnahmung der Einzelfälle im Sinne einer Klassifikation, die um der Trennschärfe zu anderen Begriffen (Klassen) willen die Besonderheiten der Einzelfälle nivelliert. Es folgt der Logik der Subsumtion des Besonderen unter das Allgemeine. Dabei geht es jedoch nicht um den Vorgang der Subsumtion selbst, sondern um den Zusatz, dass die Subsumtion der Logik des ausschließenden ,oder ${ }^{6}$ folgt: Entweder der Gegenstand fällt unter den Begriff oder er fällt nicht unter den Begriff. Die Worte Freges werfen ein Licht auf die zunächst allgemeine Verortung der neueren Logikkritik, die in Deutschland auf Nietzsche zurückgeht und im 20. Jhd. von Benjamin und Adorno, jeweils mit Akzentverschiebungen, wieder aufgegriffen wurde. Fasst man sie in der allgemeinen Formel der Destruktion des Identischen zusammen und betrachtet Freges scharfe Begriffsbegrenzung als das Verfahren, das dem identifizierenden Denken entspricht, so bedeutet Benjamins Kritik an dem logisch-mathematischen Identitätsbegriff nicht Verwerfung jeglicher Analogien, sondern Kritik an der Subsumtion verschiedener Fälle unter denselben Begriff unter Absehung ihrer individuellen Unterschiede. Dies ist der erste Gedanke, den es dabei festzuhalten gilt.

Der zweite nimmt Bezug auf die Bemerkung Adornos, dass sich ihm beim Studium der Baudelaire-Arbeiten ein Gefühl der „Künstlichkeit“ (ADORNO / BENJAMIN 1994:367) aufdränge, vor allem dort, ,wo die Arbeit anstelle der verpflichtenden Aussage die metaphorische setzt". Was Adorno mit einem negativen Vorzeichen versieht, wird von DIETER SDUN (1994:6) affirmativ gewendet: „Die Bedeutungsbreite so vieler Begriffe Benjamins entspringt dabei häufig dem Umstand, dass Benjamin zwar über einen Begriff spricht, doch im Modus des Bildes.“ Im Hinblick auf die Operationsweise der präzisierenden Eingrenzung sind zwei polare Ordnungen innerhalb der Sprache festzuhalten: die der scharfen Begriffsbegrenzung, die dem logisch-mathematischen Erkenntnisanspruch verpflichtet ist und jede Polyvalenz im Verhältnis von Bezeichnung und Ding ausschließt, und die des schillernden Zwischenraumes von Bild und Begriff bei Benjamin, in dem die exakt abgesteckten Bedeutungsbezirke von Begriffen aufgeschlossen und ihre Durchlässigkeit herbeigeführt wird. Diese zwei besagten Ordnungen begreifen - was sich nun für unsere Frage als bedeutsam erweist - zwei Formen der Einheit mit ein: 
die ,Einheit der Zahl', die die Rekurrenz von einzelnen Begriffen innerhalb eines linear verlaufenden Denkkontinuums auf seine Grundeinheit sichert ${ }^{7}$, und die Einheit der ,Konfiguration“ bei Benjamin, in der die Zusammengehörigkeit von Elementen kein begriffliches Kontinuum bildet und keine Rekurrenz aufweist, sondern sich in der „Methode als Umweg“ (BENJAMIN 1972e:208) als Bild oder Denkbild vergegenwärtigt. Folgen wir dem Gesagten, so ergibt sich für unsere Analyse ein schematischer Rahmen, nach dem der begriffliche Darstellungsmodus in der Einheit der Zahl gründet, der bildhafte dagegen der Einheit der Konstellation entspricht. Was sich nun bei Benjamin mit einer ihm eigenen Prägnanz kundtut, ist ein Ineinanderumschlagen und ein Sich-Übereinanderschieben beider Arten der Einheit. Dies sei nun an zwei Beispielen illustriert, die ich dem Traktat Der Ursprung des deutschen Trauerspiels und einer Skizze aus der Einbahnstraße entnehme. In dem Traktat heißt es:

Denn nicht an sich selbst, sondern einzig und allein in einer Zuordnung dinglicher Elemente im Begriff stellen die Ideen sich dar. Und zwar tun sie es als deren Konfiguration. Der Stab von Begriffen, welcher dem Darstellen einer Idee dient, vergegenwärtigt sie als Konfiguration von jenen. (BENJAMIN 1972e:214)

Der Forderung Benjamins, die Ideen durch eine Konfiguration von Begriffen darzustellen, liegt keine trennscharfe Scheidung von Zahl und Konfiguration zugrunde. Folgende Bemerkung SDUNs (1994:42) scheint deshalb den Sachverhalt auf den Punkt zu treffen:

Doch ist die Konfiguration eher eine Eigenschaft des Bildes als des Begriffes. Während Begriffe der Linearität der Sprache folgen und nacheinander eines nach dem anderen darstellen, wird im Bild das gleichzeitige Miteinander, die räumliche Konfiguration von Elementen deutlich.

Mit dieser von Benjamin, wie es scheint, nicht bis zuletzt intendierten Trennung beider Formen der Einheit hängt eine gewisse Regression des Geschichtsverlaufes in ein Raumhaftes zusammen, was sich im TrauerspielTraktat in den optischen Vorstellungsbildern der Ruine, der Trümmer oder der Häufung zu einem allegorischen Bild kristallisierte. In den Skizzen Über den Begriff der Geschichte wird dies zunächst begrifflich-distinktiv begründet:

Ihr Verfahren [der Universalgeschichte als Höhepunkt des Historismus - S.L.] ist additiv: sie bietet die Masse der Fakten auf, um die homogene und leere Zeit auszufüllen. Der materialistischen Geschichtsschreibung ihrerseits liegt ein kon-

7 Gleich der in der Mathematik stattfindenden Rekurrenz einer beliebigen Zahlenreihe auf ihre Grundeinheit - die Zahl ,eins'. 
struktives Prinzip zugrunde. Zum Denken gehört nicht nur die Bewegung der Gedanken, sondern ebenso ihre Stillstellung. (BENJAMIN 1972a:702)

Daraus ist zu ersehen, wie Benjamin das Additive, das dem mathematischen Vorstellungskreis angehört, dem konstruktiven Prinzip entgegensetzt, dessen konstitutives Moment eine Stillstellung und damit eine Verräumlichung des geschichtlichen Geschehens darstellt. Auf der Ebene der Sprache findet dies seinen Ausdruck darin, dass Benjamin seine durchgehend begrifflich-analytische Darstellungsweise in den Skizzen mit einer Metapher abschließt und die besagte Verräumlichung als eine nahrhafte Frucht des historisch Begriffenen bezeichnet, die einen kostbaren, ,aber des Geschmacks entratenden Samen in ihrem Inneren“ (BENJAMIN 1972a:702) habe.

Während im Trauerspiel-Traktat das Verhältnis von Zahl und Konfiguration in einem überwiegend begrifflich-analytischen Darstellungsmodus zum Vorschein kommt, so zeigt es sich an einem Beispiel aus der Einbahnstraße auf einer bildlich-assoziativen Ebene:

Zu spät gekommenes Kind. - Die Uhr im Schulhof sieht beschädigt aus durch seine Schuld. Sie steht auf ,Zu spät‘. Und in den Flur dringt aus den Klassentüren, wo es vorbeistreicht, Murmeln von geheimer Beratung. Lehrer und Schüler dahinten sind Freund. Oder es schweigt alles still, als erwarte man einen. Unhörbar legt es die Hand auf die Klinke. Die Sonne tränkt den Flecken, wo es steht. Da schändet es den grünen Tag und öffnet. Es hört die Lehrerstimme wie ein Mühlrad klappern; es steht vor dem Mahlwerk. Die klappernde Stimme behält ihren Takt, aber die Knechte werfen nun alles ab auf das neue; zehn, zwanzig schwere Säcke fliegen ihm zu, die muß er zur Bank tragen. An seinem Mäntelchen ist jeder Faden weiß bestaubt. Wie eine arme Seele um Mitternacht macht es bei jedem Schritt Getöse, und keiner sieht es. Sitzt es dann auf dem Platz, so schafft es leise bis Glockenschlag. Aber es ist kein Segen dabei. (BENJAMIN 1972c:113f.)

Das Bild stellt eine Endsituation dar, in der die Zeit stillsteht und sich in dem Bild der „,beschädigten Uhr durch seine Schuld“ in eine räumliche Vorstellung zu transformieren scheint. So wird die Uhr von dem Kind nicht als Instrument einer rein formalen, von jedem Gefühlsgehalt freien Zeitmessung wahrgenommen, sondern als Indikator seines Seelenzustands. Die Zahl als Repetition in dem optischen Bild der ,wie ein Mühlrad klappernden Lehrerstimme“ oder als Quantität im Bild des ,zehn, zwanzig schwere Säcke“ zur Bank tragenden Kindes verschmilzt hier mit der Zahl scheinbar enthobenen Vorstellungen - mit den Metaphern der Verlassenheit und der Isolation, die das Kind als einen unheilvollen Wechselrhythmus von Glück („Die Sonne tränkt den Flecken, wo es steht“), Angst (,Murmeln von geheimer Beratung“), Zögern (,Oder es schweigt alles still“), getroffener Entscheidung 
(,Unhörbar legt es die Hand auf die Klinke“), von Bemühung („Sitzt es dann auf dem Platz, so schafft es leise mit bis Glockenschlag") und ihrer unseligen Vergeblichkeit („Aber es ist kein Segen dabei.“) erfährt. Die ganze Bildsequenz exemplifiziert einen Vorgang, in dem die früher apostrophierten Einheiten der Zahl und der Konfiguration bei Benjamin eine, möchte man sagen, unmögliche Verbindung eingehen. Denn die Zahl, was hier stets als Voraussetzung meines Verfahrens gilt, bedeutet seit Anbeginn eine unbedingte Abgrenzung. ${ }^{8}$ Dies führt zu der zweiten Operationsweise hin, die dem Funktionsbegriff der Zahl zugrunde liegt und weiter oben als isolierende Abgrenzung bezeichnet wurde. Sie bedeutet eine Akzentverschiebung von dem Aspekt der Eindeutigkeit und Exaktheit auf den Aspekt der Differenz. Es sei nun ein Exkurs eingeschaltet, in dem ich im Anschluss an den Ansatz Freges und Wittgensteins auf den Begriff der Differenz näher eingehe.

Es sind zwei Arten von Differenzierung zu unterscheiden: die transitive und die nichttransitive. Eine grammatische Demonstration der ersten - die hier nur in aller Kürze rekonstruiert werden kann - findet sich bei Wittgenstein und dessen Spielverfahren, das er an einer Stelle als „Findung und Erfindung von feinen Zwischengliedern“" ${ }^{*}$ wischen Begriffen bezeichnet, die ihre trenn-

8 Dies wird bereits in der mathematischen Intuition offenbar, die dem abendländischen Zahlenverständnis zugrunde liegt. Als deren Zeugnis kann eine spätantike Nachricht gelten, die die Legende eines Griechen überliefert, der durch seine Entdeckung der irrationalen Zahlen ein göttliches Geheimnis preisgegeben habe und zur Strafe für seinen Frevel durch einen Schiffbruch umgekommen sei. In diesem sonderbaren Mythos spiegelt sich bereits eine erste ursprüngliche Einstellung zur Zahl, wie sie noch die Pythagoräer hatten, und die Furcht, dass durch das Inkommensurable irrationaler Zahlen Grenzen zwischen Dingen verloren gehen könnten. Siehe dazu den Kommentar von KURT VON FrITZ (1965:301), der in dem Zusammenhang den Namen des Hippasos von Metapont anführt: „Die Entdeckung der Inkommensurabilität muß in pythagoreischen Kreisen einen ungeheueren Eindruck gemacht haben, weil sie mit einem Schlag den Glauben zerstörte, auf dem bis dahin die ganze Philosophie der Pythagoräer beruht hatte, nämlich dass alle Dinge in ganzen Zahlen ausgedrückt werden könnten. Dieser Eindruck spiegelt sich deutlich in jenen Legenden, welche berichten, Hippasos sei von den Göttern bestraft worden, weil er diese schreckliche Entdeckung veröffentlicht habe.“

9 Vgl. Wittgenstein und seine Beschreibungen, die sich auf seine Lehre von der ,übersichtlichen Darstellung ' beziehen. „Es ist eine Hauptquelle unseres Unverständnisses, daß wir den Gebrauch unserer Wörter nicht übersehen. - Unserer Grammatik fehlt es an Übersichtlichkeit. - Die übersichtliche Darstellung vermittelt das Verständnis, welches eben darin besteht, daß wir die Zusammen- 
scharfen Grenzen aufschließe und durchlässig mache. Wittgenstein hat in seinen Philosophischen Untersuchungen aufgezeigt, dass sich der Vorgang der Differenzierung nicht nur in einem „abgeschlossenen Bezirk“ ${ }^{10}$ vollziehe, sondern auch Aufdeckung und Konstruktion von entfernten Analogien sein könne. Auf die Präsentation seines „grammatischen Verfahrens“"11 muss an dieser Stelle verzichtet werden. Es sei lediglich bemerkt, dass das analogische Denken - so wie es Wittgenstein exemplarisch dargelegt hat - die Porosität oder Offenheit der Begriffe mit einbegreift. Von der Unbestimmtheit unterscheidet sich Wittgensteins Definition poröser und offener Begriffe jedoch dadurch, dass diese immer korrigierbar und ergänzungsfähig sei, wohingegen die Unbestimmtheit nur durch genauere Regeln behoben werden könne. ${ }^{12}$ Das analogische Denken Wittgensteins differenziert also nicht durch strenge Begriffsunterscheidung (was ja gerade die Logik der scharfen Begriffsbegrenzung leistet), sondern durch feine Nuancierungen von Unterschieden. Das Unterscheiden könne sich als ein Hervorheben von feinen Analogien erweisen, weil das Analoge nicht an irgendeinem gemeinsamen Merkmal zwischen Dingen nachgewiesen wird, sondern vor dem Hintergrund einer gemeinsamen Entwicklungslinie der Fälle. So sagt Wittgenstein: „Ich kann diese Ähnlichkeiten nicht besser charakterisieren, als durch das Wort

hänge sehen. Daher die Wichtigkeit des Findens und Erfindens von Zwischengliedern." (WITTGENSTEIN 1969:345)

10 Vgl. Wittgensteins Frege-Polemik: „Frege vergleicht den Begriff mit einem Bezirk und sagt: einen unklar begrenzten Bezirk könne man überhaupt keinen Bezirk nennen. Das heißt wohl, wir können mit ihm nichts anfangen. - Aber ist es sinnlos zu sagen: ,Halte dich ungefähr hier auf!' Denk dir, ich stünde mit einem Andern auf einem Platz und sagte dies. Dabei werde ich nicht einmal irgend eine Grenze ziehen, sondern etwa mit der Hand eine zeigende Bewegung machen als zeigte ich ihm einen bestimmten Punkt.“ (WITTGENSTEIN 1969:327)

11 Wittgenstein nennt seine Denkweise grammatisch: „Es ist uns, als müßten wir die Erscheinungen durchschauen: unsere Untersuchung aber richtet sich nicht auf die Erscheinungen, sondern, wie man sagen könnte, auf die Möglichkeiten der Erscheinungen. Wir besinnen uns, heißt das, auf die Art der Aussagen, die wir über die Erscheinungen machen [...]. Unsere Betrachtung ist daher eine grammatische. Und diese Betrachtung bringt Licht in unser Problem, indem sie Missverständnisse wegräumt. Missverständnisse, die den Gebrauch von Worten betreffen; hervorgerufen, unter anderem, durch gewisse Analogien zwischen den Ausdrucksformen in verschiedenen Gebieten unserer Sprache." (WITTGENSTEIN 1969:337)

12 Vgl. die einschlägigen Überlegungen von WAISMANN (1968:156-158). 
,Familienähnlichkeiten'; denn so übergreifen und kreuzen sich die verschiedenen Ähnlichkeiten, die zwischen den Gliedern einer Familie bestehen: Wuchs, Gesichtszüge, Augenfarbe, Gang, Temperament, etc. etc."'13

Bei der zweiten, nichttransitiven Art der Differenzierung tritt an die Stelle der Transitivität die Möglichkeit einer unendlichen Erweiterung des Systems. Bei FREGE (1971:152) heißt es:

In der Entwicklung der Wissenschaft kann es vorkommen, daß ein System nicht mehr genügt, nicht so, daß es teilweise als falsch erkannt wird, sondern so, daß der berechtigte Wunsch besteht, viele Einzelheiten unter einen umfassenderen Gesichtspunkt zu stellen [...]. Das wird dann dazu führen, umfassendere, d.h. übergeordnete Begriffe und Beziehungen einzuführen. (FREGE 1971:152)

Der Vorgang der Differenzierung bewegt sich dabei auf einer Linie der Schlusskette von logischen Ableitungen, wo man bei jedem Theorem nach den Wahrheiten fragt, aus denen es erschlossen ist. „So kann der Weg der Ableitungen rückwärts verfolgt werden." Daraus ist der Unterschied zwischen der transitiven und der nichttransitiven Form der Differenzierung zu ersehen. Der Vorgang der transitiven Differenzierung bedeutet gleichzeitig Konstruktion von feinen Zwischengliedern zwischen Dingen innerhalb ihrer „Familienähnlichkeit“. So können zwei Dinge familienähnlich sein, ohne dass sie ein einziges Merkmal gemeinsam haben. Bei der nichttransitiven Differenzierung wird die lineare Kohärenz von einzelnen Ableitungen einer Schlusskette durch die Einheit der Zahl gewährleistet, die ihnen zugrunde liegt. Damit muss auch der Begriff der Ähnlichkeit - sei es zwischen den einzelnen Wahrheiten der Schlusskette oder zwischen Relationen von Ableitungen - der Forderung nach Vollständigkeit und Eindeutigkeit entsprechen. FREGE (1971:154) formuliert dies für die Definition: „So muß denn das ,stückweise Definieren' und das, was man eine stufenweise Erweiterung eines Begriffs nennt, verworfen werden. Die Definition muß auf einmal geschehen; denn solange ein Begriff nicht vollständig definiert ist, ist er nicht scharf begrenzt und kann nicht anerkannt werden." Dabei bleibt festzuhalten,

13 Wittgenstein veranschaulicht dies an den Tätigkeiten, die wir ,Spiele“ nennen. Verschiedene Spiele oder Gruppen von Spielen haben gewisse Gemeinsamkeiten miteinander, aber nicht alle Spiele die gleichen. Die gemeinsamen Merkmale sind so verteilt wie die Ähnlichkeiten, die die Mitglieder einer Familie charakterisieren: manche Mitglieder haben die gleiche Augenfarbe, manche den gleichen Wuchs, manche den gleichen Gang, aber nicht alle Mitglieder haben alle diese Merkmale gemeinsam. Deshalb nennt Wittgenstein die Ähnlichkeiten, die zwischen Begriffen, wie die, die wir als Spiele bezeichnen, bestehen, „Familienähnlichkeiten“. (WITTGENSTEIN 1969:324-325) 
dass der Aspekt der Nichttransitivität der Differenzierung einer logischen Distinktion im Bereich des Allgemeinen entspricht. Und daraus resultiert nun der Zusammenhang zwischen der Operationsweise der isolierenden Abgrenzung und der intransitiven Form der Differenzierung. Denn der Vorgang der nichttransitiven Differenzierung bedeutet auch eine unaufhebbare Trennung und Isolation zwischen den Dingen. Er geht nicht von einem exemplarischen Fall aus, der über sich selbst auf andere, ähnliche Fälle verweist. Er ist kein Hinsehen im Besonderen, sondern ein Absehen vom Besonderen.

Mit dem Vorgebrachten ist eine formal-logische Grundperspektive umrissen worden, die es erlaubt, die zuvor genannten Benjamin'schen Zahlrepräsentationen nicht nur dialektisch, in einem dichten Netz von Relationen darzustellen, sondern die Voraussetzungen ihrer Begründungen zu erhellen. Als Erstes ist festzuhalten, dass die Operationsweise der isolierenden Abgrenzung und die ihr zugehörige intransitive Differenz bei Benjamin in doppeldeutiger Beleuchtung erscheinen - in ihrer Negativität als Projektion des Grotesken, des Halluzinatorischen, des Grauens ${ }^{14}$, und als positive Wendung des Technischen, des Apparathaften in eine neue Verzauberung im „Meta-Technischen “15. Dies dokumentiert eine Stelle aus dem Kunstwerk-Aufsatz:

So wird handgreiflich, daß es eine andere Natur ist, die zu der Kamera als die zum Auge spricht. Anders vor allem so, daß an die Stelle eines vom Menschen mit Bewußtsein durchwirkten Raums ein unbewußt durchwirkter tritt. Ist es schon üblich, daß einer vom Gang der Leute, beispielsweise, sei es auch nur im Groben, sich Rechenschaft ablegt, so weiß er bestimmt nichts mehr von ihrer Haltung im Sekundenbruchteil des Ausschreitens. Ist uns schon im Groben der

14 Es ist dabei bezeichnend, dass Benjamin die Kategorie des Grauens gesondert reflektiert. Das Grauen, könnte man, seinen Gedanken folgend, sagen, ergibt sich aus dem Umstand, dass der numerischen Einheit, bei der eine Relation der Gleichung vorliegt, doch ein Inhalt (z. B. Schrecken als Seelenregung) beigelegt wird. Benjamin sagt deshalb: „Dabei zeigt sich deutlich, daß das Urphänomen des Doppels, um dazusein nicht einer Gleichheit oder Ähnlichkeit der doppelten Gegenstände bedarf, sondern daß vielmehr umgekehrt Gleichheit etwas ist, was unter der des Doppelt sich leicht einstellt. Ein Mensch kann im höchsten Schrecken dazu kommen, den nachzumachen, vor dem man erschrickt.“ In einer imaginären Verdoppelung trenne sich die Seele von dem Leib und bilde einen Strudel, bei dem , ,[...] aus allen Gliedmaßen und Bezirken des Leibes die geistigen Momente hineingezogen werden und nun den Leib depotenziert unter Abwesenheit des Geistes, also eigentlich entleibt und vielmehr nur den Körper zurücklassen“ (BENJAMIN 1984:76).

15 Vgl. hierzu die Analyse des Begriffs des Meta-Technischen und dessen Zusammenhang mit Benjamins Kategorie der ,zweiten Natur' bei LiNDNER (2006:245). 
Griff geläufig, den wir nach dem Feuerzeug oder dem Löffel tun, so wissen wir doch kaum von dem, was sich zwischen Hand und Metall dabei eigentlich abspielt, geschweige wie das mit den verschiedenen Verfassungen schwankt, in denen wir uns befinden. Hier greift die Kamera mit ihren vielen Hilfsmitteln - ihrem Stürzen und Steigen, ihrem Unterbrechen und Isolieren, ihrem Dehnen und Raffen des Ablaufs, ihrem Vergrößern und ihrem Verkleinern ein. (BENJAMIN 1980:461)

Was die Worte zur Darstellung bringen, ist eine mikrologische Art von Wahrnehmung, die sich in die kleinsten Intervalle, in die flüchtigsten Passagen zwischen den Dingen versenkt. Was sich zwischen „Hand und Metall“, „,im Sekundenbruchteil des Ausschreitens“ zu verflüchtigen droht, wird von der Kamera wieder eingesammelt und unserem Wahrnehmungsvermögen verfügbar gemacht. Die Paradoxie dieses Aktes bei Benjamin liegt nun darin, dass die Verfahrensweise der Kamera, die auf eine Fragmentierung der Welt, auf eine Isolierung ihrer einzelnen Ausschnitte angelegt sei, zugleich die Auslöschung jeglicher apparatfreier Realitätsbereiche herbeiführe. Dies bedeutet auch, dass sich die Grenze zwischen der filmtechnischen Illusion, die durch Bildmontierung vermittelt wird, und der Schwelle der als unmittelbar empfundenen Realität verflüssigt. Die Folge dieses Sachverhalts sei eine Stauung von Bildern in Kollektivträumen, in denen die Figur der MickyMaus (BENJAMIN 1980:462) in grotesker Umkehrung den Bereich des Psychotischen und Halluzinatorischen besetze. Das Halluzinatorische als ein Ineinander-Fließen, ein Ineinander-Verschwimmen von disparaten Wahrnehmungsbereichen (Moment der Identifikation) bildet hier nur die Kehrseite eines radikalen Erwachens, das auf die apparative Technik zurückgeht, durch Vergrößerung und Abgrenzung eines Details die linear-homogene Wahrnehmung zu sprengen (Moment der Isolation). So sagt BENJAMIN (1980:462): „In die alte heraklitische Wahrheit - die Wachenden haben ihre Welt gemeinsam, die Schlafenden jeder eine für sich - hat der Film eine Bresche geschlagen." Worauf es hier jedoch ankommt, ist bereits früher in der Formel ,Wendung des Technischen in eine neue Verzauberung im Meta-Technischen` zusammenfasst worden. BENJAMIN (1980:462) versucht den Verzauberungsvorgang zu begründen und sagt:

Wenn man sich davon Rechenschaft gibt, welche gefährlichen Spannungen die Technisierung mit ihren Folgen in den großen Massen erzeugt hat - Spannungen, die in kritischen Stadien psychotischen Charakter annehmen - so wird man zu der Erkenntnis kommen, daß diese selbe Technisierung gegen solche Massenpsychosen sich die Möglichkeit psychischer Impfung durch gewisse Filme geschaffen hat, in denen eine forcierte Entwicklung sadistischer Phantasien oder masochistischer Wahnvorstellungen deren natürliches und gefährliches Reifen in 
den Massen verhindern kann. Den vorzeitigen und heilsamen Ausbruch derartiger Massenpsychosen stellt das kollektive Gelächter dar.

Benjamin spricht hier dem entfremdenden und pathogenen Potenzial von technischen Bildern eine geradezu kathartische Wirkung zu. An einer anderen Stelle bringt er die Perfektibilität von technischen Bildern in direkte Verbindung mit Zahlen:

Das ist seine Verbesserungsfähigkeit. Der fertige Film ist nichts weniger als eine Schöpfung aus einem Wurf, er ist aus sehr vielen einzelnen Bildern und Bildfolgen montiert, zwischen denen der Monteur die Wahl hat - Bildern, die im übrigen von vornherein in der Folge der Aufnahmen bis zum endgültigen Gelingen beliebig zu verbessern gewesen waren. Um seine ,Opinion publique', die 3000 $\mathrm{m}$ lang ist, herzustellen, hat Chaplin $125000 \mathrm{~m}$ drehen lassen. Der Film ist also das verbesserungsfähigste Kunstwerk. (BENJAMIN 1980:446)

Auch in diesem Zitat tritt die Ambiguität der Zahl zutage, die zum einen Fragmentarisierung und Isolierung innerhalb des Bildmaterials bedeutet, zum anderen dessen Humanisierung in der Aufhebung der Isolierung durch die Montage.

Aus dem bislang Gesagten ergeben sich zwei Formen der Einheit: die Einheit der Zahl und die Einheit der Konfiguration. Die Einheit der Konfiguration entspricht der Benjamin'schen ,Methode als Umweg', die im (Denk-)Bild ihre semantische und ästhetische Realisation gefunden hat. Versuchen wir nun näher auf die Beschaffenheit und die Struktur der Benjamin'schen Einheit einzugehen.

Einen geeigneten Anhaltspunkt für unsere Frage bieten zwei kleine Schriften Benjamins: Lehre vom Ähnlichen und Über das mimetische Vermögen. Benjamins Gedankengang bewegt sich hier nicht in der mittleren Zone des abwägend-sachlich argumentierenden Verstandes, der seinen Gegenstand in einem wachen und bewussten Verstehensprozess zu umgrenzen sucht, sondern er bezieht eine, möchte man sagen, prälogische und vorkritische Randposition, wo die Natur, die Astrologie und Graphologie ihre verborgenen Korrespondenzen offenbaren und von der aus er das Phänomen der Ähnlichkeit einzukreisen versucht. Wir stoßen auf einen charakteristischen Zug in der Prosa Benjamins, dass er die Dinge nicht benennen, mit der Sprache nicht einfangen will, sondern nur auf Korrespondenzen zwischen den differenten Konstellationsbereichen (,sinnliche" Korrespondenzen innerhalb der Natur, ,unsinnliche“ Ähnlichkeiten in der Sprache des Menschen) hinweist. Das Verfahren Benjamins stellt sich dar als ein sprachlicher Nachvollzug von mimetischen Stadien, in denen die ursprüngliche Fähigkeit des Nachahmens, die beim Naturmenschen und noch beim Kind an keinen Gegenstand oder an 
keine sie vermittelnde Instanz gebunden war, in das Archiv der Sprache als ihren letzten ,unsinnlichen' Darstellungsbereich Eingang fand. BENJAMIN (1977:212) stellt die Struktur der ,unsinnlichen Ähnlichkeit` folgendermaßen dar:

Ordnet man nämlich Wörter der verschiedenen Sprachen, die ein Gleiches bedeuten, um jenes Bedeutete als ihren Mittelpunkt, so wäre zu erforschen, wie sie alle - die miteinander oft nicht die geringste Ähnlichkeit besitzen mögen ähnlich jenem Bedeuteten in ihrer Mitte sind. Jedoch ist diese Art von Ähnlichkeit nicht nur an den Verhältnissen der Wörter für Gleiches in den verschiedenen Sprachen zu erläutern.

Die Passage illustriert die charakteristische Denkbewegung bei Benjamin, die den Begriff der Ähnlichkeit über das punktuelle, linear verlaufende Äquivalenzverhältnis hinaushebt und ihm einen perspektivischen Charakter verleiht. Sie vollzieht sich in seiner Sprache als eine Sammlung einzelner Bedeutungsträger (hier Wörter, Sätze) um einen imaginären Mittelpunkt des Bedeuteten, doch so, dass die Ähnlichkeit nicht auf einer zur Deckung gelangenden Entsprechung basiert, sondern sich in der Differenz zeige, die als Möglichkeitsraum von Beziehungen aufzufassen sei. Vor dem Hintergrund - und an der Stelle ist noch einmal kurz auf die früher getroffene Unterscheidung von transitiver und intransitiver Differenz zurückzukommen - lässt sich eine Verknüpfungslinie beobachten zwischen dem Begriff der Differenz bei Benjamin und der transitiven Form der Differenz, die früher im Zusammenhang mit dem Verfahren Wittgensteins dargelegt wurde. Wir können hier darauf nicht näher eingehen. Es sei nur vermerkt, dass die transitive Form der Differenz, die eine Porosität und Durchlässigkeit der Begriffe voraussetzt, insofern als ein verbindendes Element zwischen Benjamin und Wittgenstein gelten kann, als sie mit der ihnen gemeinsamen synoptischen (übersichtlichen) Darstellungsweise zusammengeht. ${ }^{16}$

Was Benjamin besonders auszeichnet, ist nicht das Irrationalistische oder Mythologisierende mancher seiner Verfahrensweisen, sondern eine Exzentrizität der Wahrnehmung, die seine gesamte Philosophie prägt und mit der die bereits früher erwähnte Inselhaftigkeit seines Denkens zusammenhängt. Wir versuchen dies nun an einem Vergleich der zwei vorhin behandelten Schrif-

16 Darauf mögen die bekannten Aussagen beider Autoren hindeuten: „Ich habe nichts zu sagen, nur zu zeigen.“ (BENJAMIN 1982:8) Und: „Denke nicht, sondern schau." (WITTGENSTEIN 1969:66) 
ten ${ }^{17}$ mit den Fragmenten Zur Sprachphilosophie und Erkenntniskritik aufzuzeigen. Was an beiden Texten bereits bei der ersten Lektüre auffällt, ist ihre radikale Andersartigkeit in der Darstellungsweise des Phänomens der Ähnlichkeit. Der erste Text stellt ein Gefüge von Bedeutungszuweisungen dar, die ihren Gegenstand - wie bereits früher gesagt wurde - von der Peripherie her weniger durch Begriffe als durch verschiedene Bereiche von Ähnlichkeitskonstellationen (Astrologie, Kinderspiel, Graphologie, Sprache) einzukreisen versuchen. Es scheint, als ob Benjamin darin seine gesamte Darstellung des Ähnlichkeitsphänomens im Zustand einer intendierten Unschärfe belassen wollte. So bezeichnet er die zentrale Kategorie der Schrift die ,unsinnlichen Ähnlichkeiten', die er der Sprache zuweist, rätselhaft als „Verspannungen nicht nur zwischen dem Gesprochenen und Gemeinten, sondern auch zwischen dem Geschriebenen und Gemeinten und gleichfalls zwischen dem Gesprochenen und Geschriebenen“" (BENJAMIN 1977:212). Oder er überlässt sie der physiognomischen Sehweise, der eine ausgeprägt intuitivimaginäre Komponente anhaftet:

Die mit Bewußtsein wahrgenommenen Ähnlichkeiten - z.B. in Gesichtern sind verglichen mit den unzählig vielen unbewußt oder auch gar nicht wahrgenommenen Ähnlichkeiten wie der gewaltige unterseeische Block des Eisbergs im Vergleich zur kleinen Spitze, welche man aus dem Wasser ragen sieht. (BENJAMIN 1982:205)

Der Duktus des zweiten Textes ist dagegen betont begrifflich-differenzierend. Das Verfahren Benjamins stellt dort einen von klarer Vernunft geleiteten Bestimmungsversuch des Ähnlichkeitsbegriffes dar, der von dem der Analogie und der Verwandtschaft scharf abgegrenzt wird:

Analogie ist vermutlich eine metaphorische Ähnlichkeit, d.h. eine Ähnlichkeit von Relationen, während im eigentlichen Sinne (unmetaphorisch) ähnlich nur Substanzen sein können. Die Ähnlichkeit zweier Dreiecke z.B. müßte sich demgemäß als Ähnlichkeit irgendeiner Substanz an ihnen erweisen, deren Manifestation dann die Gleichheit (nicht Ähnlichkeit!) gewisser Relationen an ihnen ist. Weder aus Analogie noch aus Ähnlichkeit kann Verwandtschaft zureichend erschlossen werden; während aber die Ähnlichkeit in gewissen Fällen Verwandtschaft anzukündigen vermag, findet dies niemals in der Analogie statt. (BENJAMIN 1985:43)

Hält man nun die zwei Texte vergleichend nebeneinander, so fällt nicht nur der enge Raum auf, auf dem sich ihr Gedankengang konzentrisch eingrenzt,

17 Die zwei kleinen Schriften Lehre vom Ähnlichen und Über das mimetische Vermögen sind so angelegt, dass sie ein Textcorpus bilden. Im Folgenden werden sie somit als ein Text aufgeführt. 
sondern vor allem eine schroffe Gegensätzlichkeit ihrer Darstellungsformen. Ich habe dies früher mit dem Wort ,inselhaft' bezeichnet und damit das Moment der Abgelöstheit und Selbstbezüglichkeit beider Darstellungsformen herausgehoben. Denn zwischen der betont synoptischen Wahrnehmungsweise des ersten Textes und der begrifflich-differenzierenden des zweiten gibt es bei Benjamin keine narrativen Übergangszonen und Durchgangsstellen, die aus der doppelten Wahrnehmungsoptik, die in beiden Fällen zum Aphoristischen und Sentenziösen neigt, zu dem einsinnigen Vorgang einer essayistischen Gedankenentfaltung hinüberführen würden. ${ }^{18}$ Diesem Sachverhalt liegt auch die Tatsache zugrunde, dass das ,(Denk-)bild', das bei Benjamin auf das Narrative ganz verzichtet, zu einer genuinen, ja schicksalhaften Ausdrucksform Benjamins geworden ist: „Was an Benjamin so schwer zu verstehen war, ist, daß er, ohne ein Dichter zu sein, ,dichterisch dachte', und daß die Metapher daher für ihn das größte und geheimnisvollste Geschenk der Sprache sein mußte, weil sie in der Übertragung es möglich macht, das Unsichtbare zu versinnlichen.“ (ARENDT 1971:22) So hat auch das Disparate und Inkommensurable zwischen dem bildhaften und begrifflichen Darstellungsmodus bei Benjamin einerseits eine Vielfalt von Prosaformen ergeben, andererseits aber auch ihre stilistisch-semantische Fragmentierung und Verkürzung bewirkt, die sich selbst in seinen größeren Schriften wie dem Trauerspiel-Traktat bemerkbar macht. ${ }^{19}$ Vor diesem Hintergrund sind die Worte zu verstehen, die BENJAMIN (1966/2:556) in seinen späten Jahren an den Freund Gerhard Scholem richtet:

Die literarischen Ausdrucksformen, die mein Denken in den letzten zehn Jahren sich geschaffen hat, sind restlos bestimmt durch die Präventivmaßnahmen und Gegengifte, mit welcher ich der Zersetzung, die mich, infolge jener Kontingen-

18 Die Bezeichnung ,essayistisch“ im Zusammenhang mit dem Merkmal der Narrativität entspricht, was hier als bekannt vorausgesetzt werden darf, der klassischen Essayform, die auf Montaigne zurückgeht und in Deutschland z.B. noch von Kassner, Rychner oder Hofmannsthal gepflegt wurde. Insofern hat sie hier keinen normativen Wert und dient ausschließlich der kontextuell bedingten Argumentation. Vgl. hierzu die Kapitel bei RoHNER (1966:45-56): Bacon im Vergleich mit Montaigne und Der Begriff Essay bei den „klassischen“ deutschen Essayisten (ROHNER 1966:95-100).

19 Auf diese Disparatheit oder Differenz, die bei Benjamin den einzelnen Einheiten von Konstellationen zugrunde liegen, sind auch die Aporien von Sagbarem und Unsagbarem, von Sprechen und Zeigen zurückzuführen, die seine Philosophie kennzeichnen. Vgl. hierzu auch die akribisch durchgeführten Analysen von GABRIELLI (2004:575-664). 
zen, in meinem Denken fortgesetzt bedroht, entgegentreten mußte. So sind zwar viele, oder manche, meiner Arbeiten Siege im Kleinen gewesen, aber ihnen entsprechen die Niederlagen im Großen.

$\mathrm{Zu}$ Beginn der vorliegenden Arbeit ist ein Funktionsschema der Zahl dargestellt worden, das aus zwei Operationsweisen bestand: aus der Eingrenzung, der die Kategorien der Exaktheit und Eindeutigkeit zugeordnet wurden, und der Abgrenzung, der die Differenz entspricht. Nun ist festzuhalten, dass die ästhetisch-semantischen Zahlrepräsentationen bei Benjamin nicht immer als rein funktionelle (inhaltsfreie) Kategorien und Vorstellungsbilder fungieren, sondern stellenweise deutliche Beimengungen des Mythischen aufweisen. Betrachten wir die Art und Weise, in der er den Bereich des Mythischen handhabt, so lässt sich hierbei eine sonderbare Ambiguität bemerken. Sie geht auf die Verdoppelung der Optik zurück, in der Benjamin die Sphäre des mythischen Weltempfindens wahrnimmt. So verbindet er zwar das Mythische mit dem mimetischen Vermögen als einem zeitlichen Vorstadium der menschlichen Sprache (als Ort der Befreiung und Rettung), schreibt es aber gleichzeitig keiner früheren, von der Aufklärung als überwunden gedachten Entwicklungsperiode der Menschheit zu, sondern fasst diese als eine Art versklavender Totalität auf. So kennzeichnet auch das Mythische bei Benjamin zeitlich „die ewige Wiederkunft alles Gleichen“ (BENJAMIN 1972d:137), wie es sich auch heute noch in einem Kreislauf aus Urteil, Schuld und Unglück fortschreibe. Der funktionelle Charakter der Zahl, der der Differenz von der gemessenen und gelebten Zeit zugrunde liegt, bedeutet allerdings auch, dass die Zeit nicht stillsteht und in ein Raumhaftes übergeht und die menschliche Geschichte nicht „Unglück auf Trümmer und Trümmer auf Unglück häuft“" (BENJAMIN 1972a:697). Es gilt deswegen festzuhalten, dass vom Standpunkt der Zahl als eines Funktionsbegriffes das Gleiche nur dann wiederkehren könnte, wenn die Trennungslinie von Ewigem (Mythos) und Unendlichem (Zahl) porös, durchlässig wäre und die Grenzen zwischen beiden Ordnungen verschwimmen müssten. Die Zahl jedoch, wie sie im Vorangehenden bestimmt wurde, bedeutet eine unbedingte Abgrenzung. ${ }^{20}$ So

20 Zuvor wurde die Einheit der Zahl (im Gegensatz zu der Einheit der Konstellation bei Benjamin) dadurch charakterisiert, dass sie die Rekurrenz von einzelnen Begriffen (im Sinne der Begriffsidentitäten) innerhalb eines linear verlaufenden Denkkontinuums auf seine Grundeinheit (wie eine beliebige Zahlenreihe auf die Zahl ,eins') sichert. Die Rekurrenz ist allerdings nur dann möglich, wenn das Unendliche (eine unendliche Reihe von Begriffen innerhalb eines Denkkontinuums) als Grenze verstanden wird. Nur vor dem Hintergrund des Unendlichen als Limit kann sich eine bestimmte Zahl $(1,2,3,4)$ nicht vergrößern oder verkleinern. 
müsste sich die Wiederkehr des Gleichen im Sinne einer so aufgefassten Zahl jedesmal als Zufall erweisen. ${ }^{21}$ Mit der von Benjamin, wie es scheint, nicht immer bis zuletzt vollzogenen Trennung der mythischen Ordnung von der mathematisch-logischen der Zahl verbindet sich jene bereits früher angedeutete Unbestimmtheit, auf die auch MENNINGHAUS (1986:10) hinweist:

Und das Benjaminsche Verfahren der Bedeutungszuweisung qua Konstellation gleitet gerade beim Mythos-Begriff nicht selten aus einem produktiven Spiel mit Unschärfen, die sich kontextuell illuminieren, in schlichte semantische Unbestimmtheit ab, deren Mangel kraft sprachlicher Emphase dann auch teilweise noch in den Schein besonderer ,Tiefe" umgebogen wird.

Der Grund für die besagte Unbestimmtheit liegt darin, dass die Dimension der Zeit durch ihre Verräumlichung bei Benjamin gleichsam in das Bild (als ein genuin Raumhaftes) mit einfließt, womit Zeit und Geschichte gleichermaBen ihre Einsinnigkeit verlieren, was sich auf der Ebene der Sprache in signifikanter Weise in dem bereits früher apostrophierten Verlust des Narrativen kundtut. ${ }^{22}$ So war denn auch Benjamins Denken weniger darauf ausgerichtet, seinen Gegenstand zu ,treffen', als ihn vielmehr einzukreisen, auf ihn nur hinzuweisen. Benjamin stellte deswegen das Ding weniger in flüssigem Lebendigsein, im Vorgang der Entfaltung dar als vielmehr im „Bild als der Dialektik im Stillstand“ (BENJAMIN 1982:578), in dem, was MAX RYCHNER (1952:232) als seine „tiefe Dialektik“ bezeichnet hat.

\section{Literatur}

AdORNO, THEODOR W. (1970): Über Walter Benjamin. Frankfurt (M.).

AdORno, THEOdOR W. / Benjamin, WALTER (1994): Briefwechsel. Frankfurt (M.).

Arendt, Hannah (1971): Benjamin, Brecht. Zwei Essays. München.

Benjamin, Walter (1966): Briefe. Bd. 2. Frankfurt (M.).

Benjamin, WaLter (1972-1989): Gesammelte Schriften. Hrsg. von Rolf Tiedemann und Hermann Schweppenhäuser. Frankfurt (M.):

21 Es sei denn, es gelte die Vorstellung, dass die unendliche Linie eine Krümmung aufweise und so in ihrem unendlichen Verlauf zu einem Kreis werde. Die Zeit würde dann zu einer vierten Dimension des Raumes werden und das Gleiche könnte wiederkehren (als Traum).

22 Dies wiederum hat in den für Benjamin charakteristischen Vorstellungsbildern der Diskontinuität seinen Niederschlag gefunden: z. B. als ,Aufblitzen“ der Wahrheit in der Kontingenz der Sprache, als ,Zusammenschießen' disparater Elemente zu einem dialektischen Bild, als ,Aufsprengen“ der geschlossenen Sinneinheit der homogenen Geschichte usw. 
- (1972a): Über den Begriff der Geschichte.

- (1972b): Über einige Motive bei Baudelaire.

- (1972c): Einbahnstraße.

- (1972d): Goethes Wahlverwandtschaften.

- (1972e): Der Ursprung des deutschen Trauerspiels.

- (1972f): Erkenntnistheoretisches, Theorie des Fortschritts.

- (1977): Über das mimetische Vermögen.

- (1980): Das Kunstwerk im Zeitalter seiner technischen Reproduzierbarkeit.

- (1982): Das Passagen-Werk.

- (1982): Lehre vom Ähnlichen.

- (1984): Zur Moral und Anthropologie. Über das Grauen.

- (1985): Zur Sprachphilosophie und Erkenntniskritik.

FREGE, GOTTLOB (1971): Schriften zur Logik und Sprachphilosophie. Hamburg.

FRITZ, KURT VON (1965): Die Endeckung der Inkommensurabilität durch Hippasos von Metapont. In: BECKER, OSKAR (ed.): Zur Geschichte der griechischen Mathematik. Darmstadt.

GABRIELLI, PAOlo (2004): Sinn und Bild bei Wittgenstein und Benjamin. Bern.

HABERMAS, JÜRGEN (1981): Walter Benjamin, bewußtmachende oder rettende Kritik. In: Habermas, JÜrgen: Philosophisch-politische Profile. Frankfurt (M.), 336-376.

LINDNER, Burkhardt (2006): Das Kunstwerk im Zeitalter seiner technischen Reproduzierbarkeit. In: LINDNER, BURGHARD (ed.): Benjamin Handbuch. Stuttgart, 229-251.

Menninghaus, Winfried (1986): Schwellenkunde. Walter Benjamins Passage des Mythos. Frankfurt (M.).

ROHNER, LuDWIG (1966): Der deutsche Essay. Materialien zur Geschichte und Ästhetik einer literarischen Gattung. Neuwied/Berlin.

RYCHNER, MAX (1952): Walter Benjamin: Nachgelassene Prosastücke. Sphären der Bücherwelt. Zürich.

SCHÄFER, LOTHAR (1974): Handbuch philosophischer Grundbegriffe. Hrsg. von Hermann Krings, Hans Michal Baumgartner und Christoph Wild. München, 1775-1787.

SDUN, DiETER (1994): Benjamins Käfer. Untersuchungen zur bildlichen Sprache Walter Benjamins im Umkreis der „Einbahnstraße“. Frankfurt (M.).

WAISMANN, FRIEDRICH (1968): Verifizierbarkeit. In: BUBNER, RÜDIGER (ed.): Sprache und Analysis. Texte zur englischen Philosophie der Gegenwart. Göttingen, 154-169.

WiEDMANN, FrANZ (1988): Anschauliche Wirklichkeit, Bilder und Sprachen. Würzburg. WitTGENSTEIn, Ludwig (1969): Philosophische Untersuchungen. In: WitTGENSTEIN, LuDWIG: Schriften 1. Frankfurt (M.), 15-370. 